Received: 26.09 .2018

Revised: 25.10 .2018

Accepted: 02.11.2018

DOI: $10.17804 / 2410-9908.2018 .6 .157-164$

\title{
SEPARATION OF A SOLID SOLUTION OF A Fe-Ni INVAR ALLOY WITH DIFFERENT TYPES OF POINT DEFECT SINKS UNDER ELECTRON IRRADIATION
}

\author{
S. E. Danilov ${ }^{\text {a) }}$ and V. L. Arbuzov ${ }^{\text {b) }}$ \\ M.N. Miheev Institute of Metal Physics, Ural Branch of the Russian Academy of Sciences, \\ 18 S. Kovalevskoy St., Ekaterinburg, 620990, Russian Federation \\ a) (iD https://orcid.org/0000-0001-9815-8196 danilov@imp.uran.ru; \\ b) iD https://orcid.org/0000-0003-0334-7459 arbuzov@imp.uran.ru \\ *Corresponding author. E-mail: danilov@imp.uran.ru \\ Address for correspondence: ul. S. Kovalevskoy, 18, Ekaterinburg, 620990, Russian Federation \\ Tel.: +73433783850
}

Solid solution separation is studied for electron irradiation of a Fe-Ni alloy with different types of sinks of point defects in the form of dislocations and deformation-induced vacancy clusters. It is shown that the efficiency of deformation-induced dislocation sinks exceeds that of deformation-induced vacancy clusters. In isochronous annealing, two sub-stages of dissociation of vacancy clusters with dissociation energies of 1.2 and $1.5 \mathrm{eV}$ are observed, which are independent of the method of creating vacancy clusters - deformation or electron irradiation.

Keywords: irradiation, electrons, Fe-Ni Invar, deformation, sinks of point defects, solid solution separation.

\section{Acknowledgment}

The work was performed within the state assignment on the subject of Spin, No. AAAA-A18118020290104-2.

\section{References}

1. Dimitrov C., Dimitrov O. Composition dependence of defect properties in electronirradiated Fe-Cr-Ni solid solutions. Journal of Physics F: Metal Physics, vol. 14, no. 4, pp. 793-811. DOI: $\underline{10.1088 / 0305-4608 / 14 / 4 / 005}$.

2. Mantl S., Sharma B.D., Antesberger G. Positron annihilation studies on electron- and a-particle- irradiated 75Ni13Cr12Fe alloys. Phil. Mag. A, 1979, vol. 39, no. 4, pp. 389-397. DOI: $10.1080 / 01418617908239279$.

3. Aliev S.S., Gruzin P.L., Menshikov A.Z., Mogutnov B.M., Rodionov Yu.L., Shaposhnikov N.G. Low-temperature phase transformations in iron-nickel alloys under electron irradiation. Metallophysika, 1985, vol. 7, no. 5, pp. 80-86.

4. Chamberod A., Laugier J., Penisson J.M. Electron irradiation effects on iron-nickel invar alloys. Journal of Magnetism and Magnetic Materials, 1979, vol. 10, pp. 139-144. DOI: 10.1016/0304-8853(79)90165-3.

5. Arbuzov V.L., Druzhkov A.P., Danilov S.E. Effect of phosphorous on defects accumulation and annealing in electron-irradiated Fe-Ni austenitic alloys. J. Nucl. Mater., 2001, vol. 295, iss. 2-3, pp. 273-280. DOI: 10.1016/S0022-3115(01)00505-0. 
6. Danilov S.E., Arbuzov V.L., Pecherkina N.L., Sagaradze V.V. Separation of radiation defects in deformed nickel. The Physics of Metals and Metallography, 2015, vol. 116, no. 7, pp. 711-717. DOI: 10.1134/S0031918X15070042.

7. Druzhkov A.P., Arbuzov V.L., Perminov D.A. Accumulation and Annealing of Radiation Defects in Deformed Austenitic Alloys. The Physics of Metals and Metallography, 2002, vol. 94, no. 1. pp. 68-72.

8. Arbuzov V.L., Danilov S.E., Druzhkov A.P., Perminov D.A. The Influence of Plastic Deformation and Radiation Defects on the Structural and Phase Transformations of N36 and N36T2 Austenitic Alloys. The Physics of Metals and Metallography, 2004, vol. 98, no. 5, pp. 500-505.

9. $\quad$ Kubaschewski O. Iron-Binary Phase Diagrams, Springer-Verlag, Berlin, 1982, 185 p. 
Подана в журнал: 26.09 .2018

УДК 539.12.04:621.78: 669.15.24

DOI: $10.17804 / 2410-9908.2018 .6 .157-164$

\title{
РАССЛОЕНИЕ ТВЕРДОГО РАСТВОРА ИНВАРНОГО СПЛАВА FЕ-NІ С РАЗНЫМИ ТИПАМИ СТОКОВ ТОЧЕЧНЫХ ДЕФЕКТОВ ПРИ ЭЛЕКТРОННОМ ОБЛУЧЕНИИ
}

\author{
С. Е. Данилов ${ }^{\text {a) }}$, В. Л. Арбузов ${ }^{\text {() }}$ \\ Федеральное государственное бюджетное учреждение науки \\ Институт физики металлов им. М.Н. Михеева Уральского отделения Российской академии наук, \\ ул. С. Ковалевской, 18, Екатеринбург, Российская Федерациия \\ a) (iD https://orcid.org/0000-0001-9815-8196 danilov@imp.uran.ru; \\ б) iD https://orcid.org/0000-0003-0334-7459 arbuzov@imp.uran.ru \\ *Ответственный автор. E-mail: danilov@imp.uran.ru \\ Адрес для переписки: ул. С. Ковалевской, 18, 620990, Екатеринбург, Российская Федерация \\ Тел.: +7 (343) 378-38-50
}

Исследовано расслоение твердого раствора при электронном облучении в $\mathrm{Fe}-\mathrm{Ni}$ сплаве с разными типами стоков для точечных дефектов в виде дислокаций и деформационных вакансионных кластеров. Показано, что эффективность деформационных дислокационных стоков больше, чем эффективность деформационных вакансионных кластеров. При изохронных отжигах наблюдается две подстадии диссоциации вакансионных кластеров с энергиями диссоциации 1,2 и 1,5 эВ, которые не зависят от способа создания вакансионных кластеров: деформацией или облучением электронами.

Ключевые слова: облучение, электроны, Fe-Ni инвар, деформация, стоки точечных дефектов, расслоение твердого раствора.

\section{1. Введение}

В сталях и сплавах в отличие от чистых металлов под действием облучения развиваются различные радиационно-индуцированные структурно-фазовые превращения (СФП), которые собственно и определяют изменения свойств материалов в процессе облучения и во многом определяют их радиационную повреждаемость. Эти процессы непосредственно связаны со свободной миграцией точечных дефектов, генерируемых облучением. При электронном облучении генерируются только свободно мигрирующие дефекты, которые при своей миграции образуют вакансионные кластеры. Диффузионная длина мигрирующих радиационных дефектов, определяющая эффективность облучения в осуществлении СФП, зависит от типа и концентрации стоков точечных дефектов, которыми могут служить дислокации, вакансионные кластеры, образованные на этапе приготовления, межфазные границы.

В широко использующихся аустенитных хромоникелевых сталях при облучении может происходить расслоение твердого раствора $[1,2]$. Эксперименты на модельных железоникелевых сплавах показали, что при облучении происходит расслоение на две фазы различного состава - FeNi и $\mathrm{Fe}_{3} \mathrm{Ni}$ - и при дальнейшем облучении наблюдается упорядочение в этих фазах с образованием соединений $[3,4]$. При таком расслоении прослеживается сильный рост электросопротивления, который может быть использован в качестве индикатора происходящего расслоения [5].

Целью данной работы является выяснение влияния различных типов стоков на процесс расслоения твердого раствора при электронном облучении модельного сплава $\mathrm{Fe}-\mathrm{Ni}$. 


\section{2. Методика}

Исследования были проведены на сплаве $\mathrm{Fe}-34,7 \mathrm{at.} \% \mathrm{Ni}$, содержащем около 0,01 ат.\% углерода, закаленном от $1323 \mathrm{~K}(3)$. Из этого сплава пластической деформацией $(\varepsilon=40 \%)$ был получен сплав с высокой концентрацией дислокационных стоков и вакансионных кластеров (Д) и путем отжига при 573 К после деформации - сплав, содержащий только дислокационные стоки (ДО).

Облучения 5 МэВ электронами были проведены при температуре 320 К. Образцы отжигали изохронно со средней скоростью нагрева $1 \mathrm{~K} /$ мин.

В данной работе использовалось измерение остаточного электросопротивления, чувствительного к искажениям решетки не только около дефектов, но и к изменениям порядка во взаимном расположении атомов компонентов сплава в решетке. Остаточное электросопротивление измеряли стандартным четырехзондовым потенциометрическим методом с чувствительностью на уровне 0.01 нОм см и точностью не хуже $0.05 \%$.

\section{3. Результаты и обсуждение}

При температуре облучения 320 К, выбранной в данной работе, вакансии в рассматриваемых сплавах подвижны и обеспечивают при своей миграции процессы расслоения [5]. При этом они аннигилируют на стоках, взаимодействуя друг с другом, образуют вакансионные кластеры (ВК) и участвуют в рекомбинации с межузельными атомами.

На рис. 1 приведены результаты облучения электронами. Видно, что деформация приводит к ослаблению процессов расслоения примерно в 5 раз для сплава Д и в 4 раза для сплава ДО по сравнению со сплавом 3, в котором концентрация стоков минимальна. С увеличением дозы облучения эти коэффициенты практически не меняются, что говорит о неизменном влиянии дислокационных стоков. Влияние созданных при деформации вакансионных кластеров незначительно.

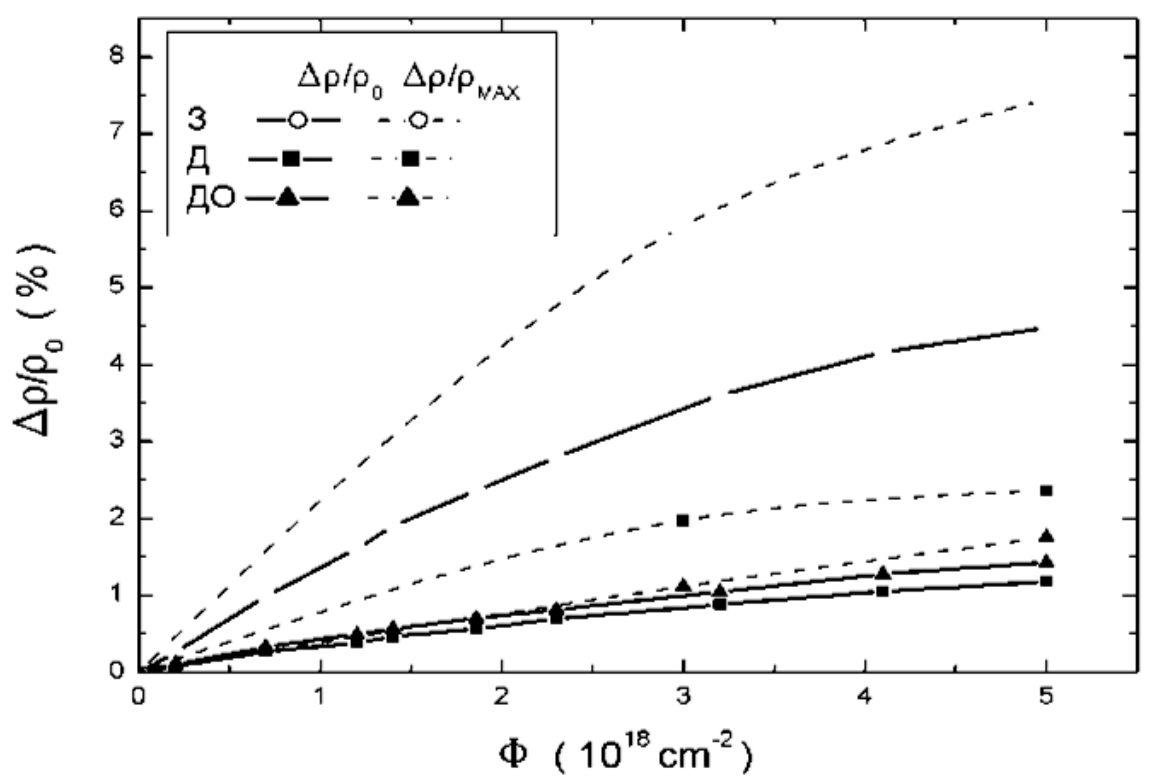

Рис. 1. Относительный прирост электросопротивления сплавов при облучении электронами при 320 К. Пунктиром показаны максимальные значения прироста, достигаемые при изохронном отжиге в районе $700 \mathrm{~K}$

Можно оценить среднюю эффективность стоков по осуществлению процессов расслоения. Для дислокаций эффективность $k_{d}^{2}=Z \rho_{d}$, где $Z$ - коэффициент, учитывающий раз- 
личие для разных типов точечных дефектов (принимаем равным 1); $\rho_{d}$ - плотность дислокаций принимаем равной $5 \cdot 10^{10} \mathrm{~cm}^{-2}[6]$. Получаем $k_{d}^{2}=5 \cdot 10^{10} \mathrm{~cm}^{-2}$. Для деформационных ВК эффективность $k_{V}{ }^{2}=4 \pi r_{V} N_{V}$, где $r_{V}$ - радиус ВК, а $N_{V}$ - концентрация ВК этого типа. Таким образом, в сплаве Д, где существуют как дислокации, так и ВК, суммарная эффективность действия стоков равна $k_{d}^{2}+k_{V}^{2}$, а для сплава ДО - только $k_{d}^{2}$. Таким образом, считая, что эффективность влияния стоков пропорциональна диффузионной длине свободного пробега вакансий и что скорость рекомбинации приблизительно одинакова, из соотношения эффективностей влияния стоков в этих сплавах при облучении (см. выше) получаем эффективность ВК $k_{V}{ }^{2}=10^{10} \mathrm{~cm}^{-2}$. Такая эффективность деформационных вакансионных кластеров соответствует концентрации ВК. Естественно, что в процессе облучения, в результате миграции свободных вакансий и их встречи друг с другом также образуются ВК. Но, например, в сплаве ДО образуется в несколько раз меньше ВК, чем в сплаве 3 [7].

С целью выяснения различия в дефектной структуре, образующейся при облучении, в сплавах различного типа проводились изохронные отжиги облученных и необлученных сталей.

Изохронные отжиги необлученных сплавов были проведены для выяснения особенностей дефектной структуры и структурно-фазовых изменений при участии деформационных ВК. Полученные результаты по изменениям сопротивления изображены на рис. 2, в нижней части графика - дифференцированные зависимости.

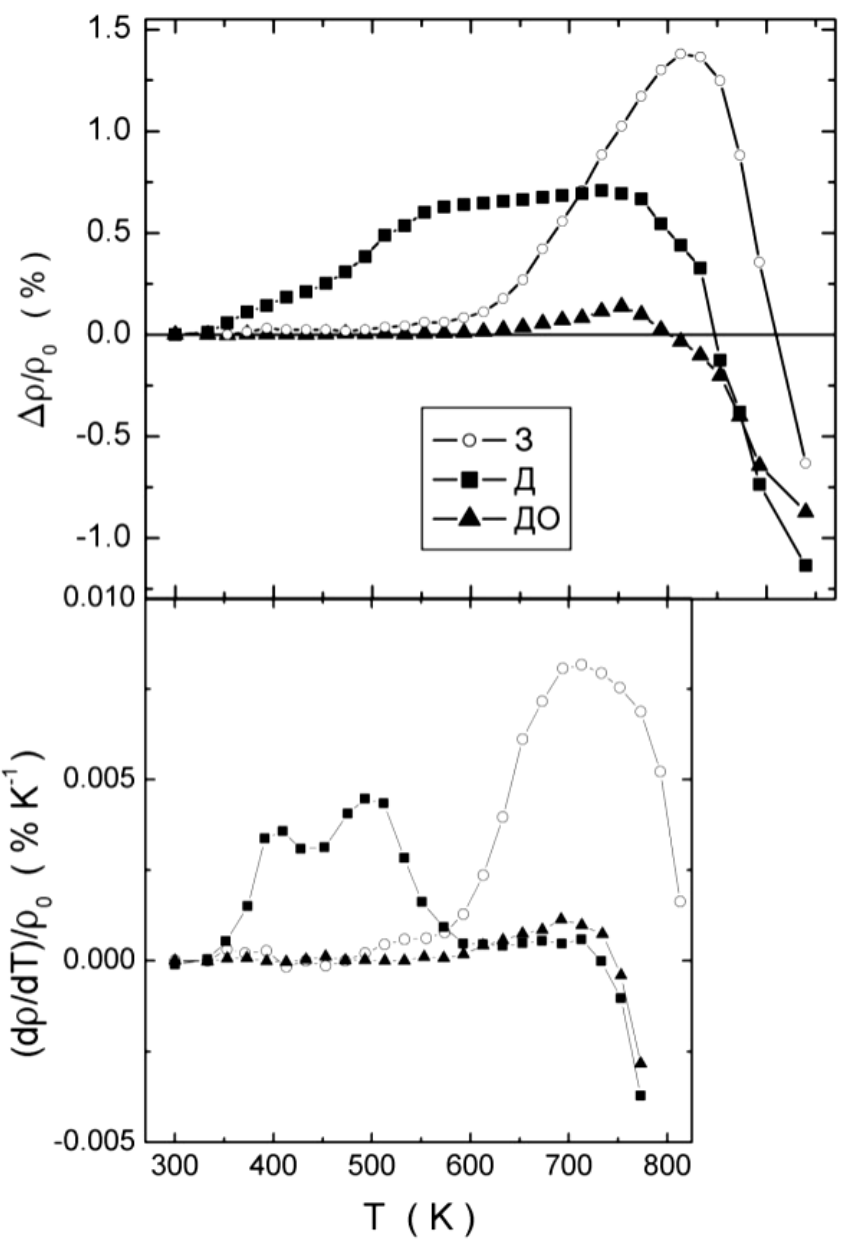

Рис. 2. Результаты изохронного отжига необлученных сталей. Нижний график дифференцированные кривые 
Как и предполагалось, диссоциация деформационных ВК приводит к росту электросопротивления начиная с 400 К в сплаве Д. Из дифференцированной кривой видно, что пик этого процесса наблюдается около $500 \mathrm{~K}$. В сплаве ДО, где для устранения деформационных ВК был проведен отжиг при 573 К, а также в сплаве 3, как и следовало ожидать, ничего не происходит до температур $750 \ldots 780 \mathrm{~K}$, когда в соответствии с полученными нами в работе [8] данными начинается отжиг дислокационной структуры в этом сплаве. В закаленном сплаве 3 сопротивление не меняется до $680 \mathrm{~K}$, когда усиление термической диффузии обусловливает процессы гомогенизации либо образование фазы $\alpha-F e$ и последующее ее растворение $[4,9]$.

На рис. 3 показаны результаты изохронного отжига для облученных электронами сплавов. В нижней части рисунка приведены дифференцированные зависимости.

Из этого рисунка видно, что отжиг после облучения приводит к дальнейшему росту электросопротивления начиная с 350 K во всех трех сплавах с максимумом роста около 480 К, причем этот рост наиболее значителен в закаленном сплаве. В сплаве Д рост больше, чем в сплаве ДО. Эти особенности подтверждают, что в этом интервале температур происходит диссоциация вакансионных кластеров. В сплаве ДО происходит только диссоциация ВК, образовавшихся при облучении, а в сплаве Д к этому добавляется диссоциация ВК, созданных деформацией. Однако действие освобождающихся вакансий на расслоение твердого раствора в сплавах Д и ДО происходит на фоне высокой концентрации дислокационных стоков, что ограничивает путь миграции вакансий. Существенно больший эффект наблюдается в сплаве 3, где концентрация дислокаций существенно ниже, и путь миграции вакансий существенно больше.

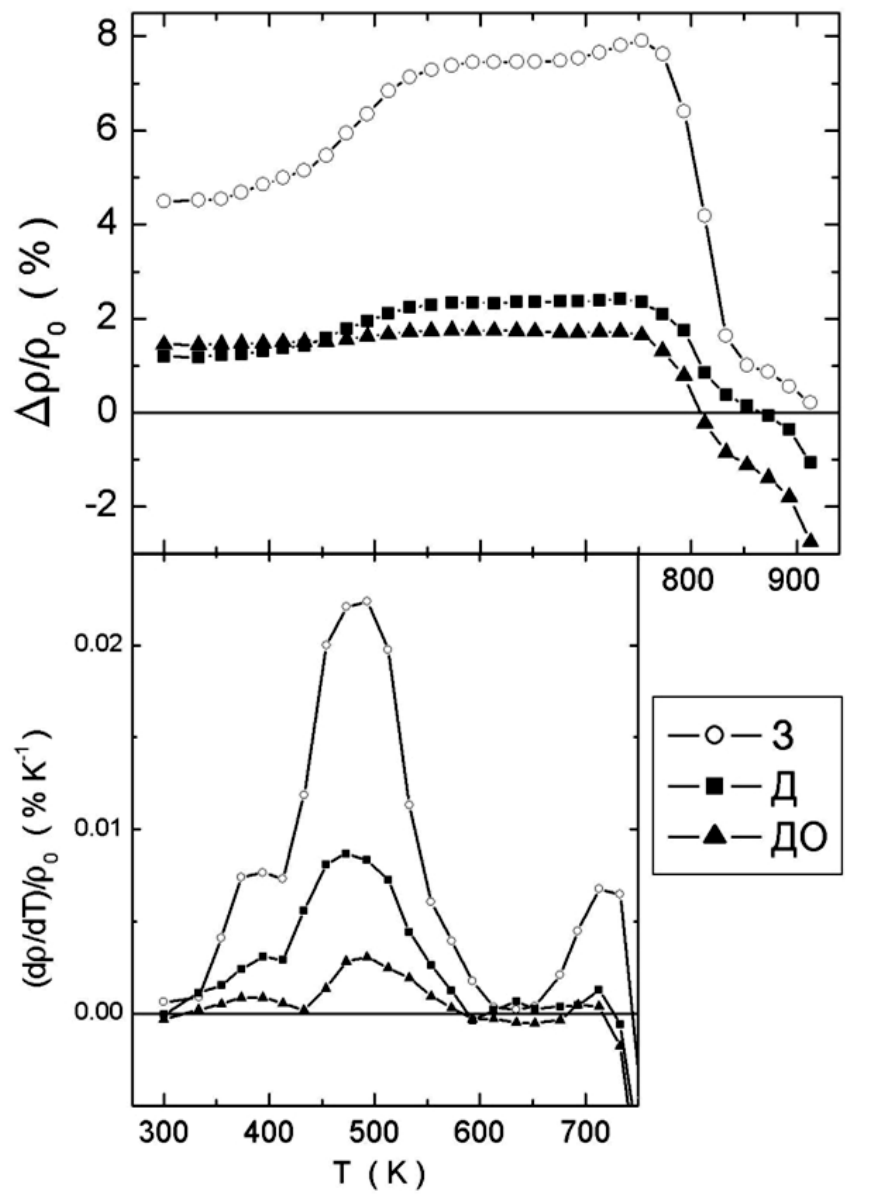

Рис. 3. Результаты изохронного отжига облученных электронами сталей.

В нижней части рисунка - дифференцированные кривые

Danilov S. E., Arbuzov V. L. Separation of a solid solution of a Fe-Ni invar alloy with different types of point defect sinks under electron irradiation // Diagnostics, Resource and Mechanics of materials and structures. - 2018. - Iss. 6. - P. 157-164. - 
По величине прироста электросопротивления на этом этапе, сравнивая с данными, приведенными на рис. 3, можно оценить количество вакансий, освобождающихся при диссоциации в сплаве Д и ДО. Такое сравнение дает, что количество освобождающихся при диссоциации вакансий в облученном сплаве Д примерно в 4 раза больше, чем в облученном сплаве ДО, и при этом в 2 раза больше количества вакансий, освобождающихся при диссоциации в необлученном сплаве Д. Из этого следует, что накопление вакансий при облучении в сплаве Д больше примерно в 2 раза, чем в сплаве ДО. Следовательно, деформационные ВК в процессе облучения работают как внутренние стоки, и на них происходит накопление генерированных облучением вакансий.

Из дифференцированных кривых на рис. 4 видно, что кроме основного пика в районе 480 К есть небольшой пик при 380 К, который присутствует также и при отжиге необлученного сплава Д. Таким образом, структура ВК не зависит от способа их создания и наличия стоков. Можно оценить энергии диссоциации ВК, соответствующие этим пикам. Она составляет 1,2 эВ для пика при 380 К и 1,5 эВ для пика при 480 К.

На облученном сплаве 3, так же как и в необлученном, присутствует пик при 700 К, и его величина примерно та же. Следовательно, он не связан с облучением и определяется, как и говорилось раньше, термической диффузией и фазовой диаграммой.

На рис. 1 показаны дозовые зависимости максимальных значений прироста электросопротивления в процессе изохронных отжигов (пунктир). С увеличением дозы облучения электронами максимальное значение для всех трех сплавов увеличивается, причем величина $\Delta \rho_{\text {мах }}-\Delta \rho_{0}$ также увеличивается, что указывает на то, что число сохранившихся при облучении вакансий в виде ВК увеличивается. Необходимо отметить, что ослабление суммарного (при облучении и при отжиге) эффекта в сплавах Д и ДО относительно 3 составляет 3,5 и 4,7 соответственно. Это отвечает механизму диссоциации при отжиге облученных электронами сплавов, описанному выше.

\section{4. Выводы}

Из полученных результатов следует, что в процессе электронного облучения дислокации и деформационные вакансионные кластеры работают как стоки для радиационных дефектов, причем эффективность дислокационных стоков больше.

При изохронных отжигах облученных и деформированных сплавов наблюдается две подстадии диссоциации вакансионных кластеров с энергиями диссоциации 1,2 и 1,5 эВ. Эти энергии не зависят от способа создания вакансионных кластеров (деформацией или облучением электронами).

Эффективность свободно мигрирующих вакансий в осуществлении процессов расслоения как при облучении так и при диссоциации ВК в процессе отжига ослабляется в 4 раза при наличии стоков в виде деформационных дислокаций.

\section{Благодарность}

Работа выполнена в рамках государственного задания по теме «Спин» №AAAA-A18118020290104-2.

\section{Литература}

1. Dimitrov C., Dimitrov O. Composition dependence of defect properties in electronirradiated Fe-Cr-Ni solid solutions // Journal of Physics F: Metal Physics. - Vol. 14, no. 4. -

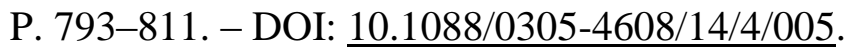

2. Mantl S., Sharma B. D., Antesberger G. Positron annihilation studies on electron- and $\alpha-$ particle- irradiated 75Ni13Cr12Fe alloys // Phil. Mag. A. - 1979. - Vol. 39, no. 4. - P. 389-397. DOI: $10.1080 / 01418617908239279$. 
3. Low-temperature phase transformations in iron-nickel alloys under electron irradiation / S. S. Aliev, P. L. Gruzin, A. Z. Menshikov, B. M. Mogutnov, Yu. L. Rodionov, N. G. Shaposhnikov // Metallophysika. - 1985. - Vol. 7, no. 5. - P. 80-86.

4. Chamberod A., Laugier J., Penisson J. M. Electron irradiation effects on iron-nickel invar alloys // Journal of Magnetism and Magnetic Materials. - 1979. - Vol. 10. - P. 139-144. DOI: 10.1016/0304-8853(79)90165-3.

5. Arbuzov V. L., Druzhkov A. P., Danilov S. E. Effect of phosphorous on defects accumulation and annealing in electron-irradiated Fe-Ni austenitic alloys // J. Nucl. Mater. 2001. - Vol. 295, iss. 2-3. - P. 273-280. - DOI: 10.1016/S0022-3115(01)00505-0.

6. Separation of radiation defects in deformed nickel / S. E. Danilov, V. L. Arbuzov, N. L. Pecherkina, V. V. Sagaradze // The Physics of Metals and Metallography. - 2015. - Vol. 116, no. 7. - P. 711-717. - DOI: 10.1134/S0031918X15070042.

7. Druzhkov A. P., Arbuzov V. L., Perminov D. A. Accumulation and Annealing of Radiation Defects in Deformed Austenitic Alloys // The Physics of Metals and Metallography. - 2002. Vol. 94, no. 1. - P. 68-72.

8. The Influence of Plastic Deformation and Radiation Defects on the Structural and Phase Transformations of N36 and N36T2 Austenitic Alloys / V. L. Arbuzov, S. E. Danilov, A. P. Druzhkov, D. A. Perminov // The Physics of Metals and Metallography. - 2004. - Vol. 98, no. 5. - P. 500-505.

9. Кубашевски О. Диаграммы состояния двойных систем на основе железа : справочник / пер. с англ. - М. : «Металлургия», 1985. - 183 с. 